\title{
INVESTIGACIONES
}

\section{Actitudes hacia la diversidad del alumnado en Educación Infantil}

\author{
Attitudes toward the diversity of students in Preschool Education
}

\author{
María Ángeles Cano-Muñoz ${ }^{a}$, María Isabel Gómez-Núñez
}

${ }^{a}$ Universidad Católica de Murcia. macano@ucam.edu

${ }^{b}$ Universidad Internacional de la Rioja. mariaisabel.gomez@unir.net

\begin{abstract}
RESUMEN
Las intervenciones educativas de carácter inclusivo con niños con necesidades específicas de apoyo educativo en la etapa de Educación Infantil se consolidan como una de las actuaciones más eficaces. Por esto, el objetivo del presente trabajo fue conocer cómo se desarrollaba la atención educativa a la diversidad en el segundo ciclo de Educación Infantil. Para esto, se optó por un diseño descriptivo simple y se empleó como instrumento de medida un cuestionario. La muestra estuvo formada por 142 maestros del segundo ciclo de Educación Infantil de diversos colegios públicos y concertados españoles. Los resultados mostraron actitudes muy positivas hacia la diversidad, poniendo en práctica medidas inclusivas para atender al alumnado. Entre las necesidades más importantes destacó el aumento de recursos personales y materiales en los centros. La implementación de prácticas educativas que atiendan a la diversidad favorece la mejora de las relaciones interpersonales y la competencia escolar.
\end{abstract}

Palabras claves: inclusión, infancia, creencias, necesidades, diversidad.

\begin{abstract}
Educational inclusive interventions with children with specific needs for educational support in the Preschool Education are consolidated as one of the most effective actions. Therefore, the aim of this work was to know how diversity educational attention was developed in the Preschool Education. To do this, a simple descriptive design was chosen and a questionnaire was used as a measuring instrument. The sample consisted of 142 teachers of Preschool Education from several Spanish public and private schools. The results showed very positive attitudes towards diversity, putting into practice inclusive measures to attend to students. Among the most important needs was the increase of personal and material resources at school. The implementation of educational practices which deal with diversity contribute to the improvement of interpersonal relationships and school competence.
\end{abstract}

Key words: inclusion, childhood, beliefs, needs, diversity. 


\section{INTRODUCCIÓN}

El término inclusión en educación es entendido como una respuesta a la diversidad de las necesidades educativas de los alumnos, que tiene en cuenta a todos y cada uno de los estudiantes, con una mayor atención a aquellos que presentan riesgo de exclusión. Así mismo, este proceso implica a toda la comunidad educativa, considerando el aula como un espacio de diálogo para tratar de favorecer la construcción de una sociedad más inclusiva, tolerante y justa (Ainscow, 2012). Sin embargo, a pesar de la extensa literatura existente sobre la inclusión del alumnado en las etapas de Educación Primaria o Educación Secundaria, la inclusión de niños con necesidades específicas de apoyo educativo en la etapa de Educación Infantil es un fenómeno de estudio relativamente reciente (Barton y Smith, 2015). De acuerdo con la legislación española actual (Ley Orgánica 2/2006, de 3 de mayo, de Educación, modificada por el artículo único de la Ley Orgánica 8/2013, de 9 de diciembre, para la mejora de la calidad educativa), se entiende por alumnado con necesidad específica de apoyo educativo aquellos que presenten necesidades educativas especiales, altas capacidades, integración tardía, condiciones personales y/o de historia escolar, dificultades específicas de aprendizaje y TDAH.

Los continuos cambios que se están desarrollando en la sociedad actual demandan que, desde los centros educativos, se planifiquen actuaciones preventivas y que se actúe de manera temprana ante las necesidades detectadas, lo que confiere a la Educación Infantil un marcado carácter proactivo. Los primeros años de vida de un niño constituyen una fase especialmente crítica, ya que en esta se van a configurar las habilidades perceptivas, motrices, cognitivas, lingüísticas y sociales que posibilitarán los aprendizajes posteriores y la interacción con el entorno (Amar, 2015), de ahí la importancia de las actuaciones que se desarrollen en esta etapa educativa.

A lo largo de los últimos años, han surgido una gran variedad de modelos educativos para la intervención con niños con necesidades específicas de apoyo educativo, tanto en Educación Infantil como en Educación Primaria (Odom, Buysse y Soukakou, 2011). Sin embargo, es preciso defender un modelo integral de actuación para favorecer el desarrollo del niño mediante acciones no solo con este, sino también con su familia y el entorno abogando, de esta forma, por una perspectiva más preventiva que terapéutica, inclusiva y, en definitiva, educativa (Giné, Balcells-Balcells, Cañadas y Paniagua, 2016; Aboes Vercelli, Alcãntara y Silveira Barbosa, 2019). Este modelo integral se concreta en distintas actuaciones de carácter inclusivo que pueden ser llevadas a cabo en los centros educativos con los alumnos, dependiendo de sus necesidades y del contexto. Siguiendo esta línea, Balongo-González y Mérida-Serrano (2016) llevaron a cabo un estudio centrado en los beneficios de la aplicación de la metodología de trabajo por proyectos en Educación Infantil. Los resultados mostraron un incremento en los niveles de inclusión en el aula debido a la ayuda entre iguales, el aumento de la motivación, la implicación emocional, la atención individualizada y la relación alumnos-docentes. Otras prácticas de carácter inclusivo llevadas a cabo en Educación Primaria y que podrían desarrollarse en Educación Infantil, vendrían definidas por la coenseñanza con más de un docente en el aula, el trabajo por rincones, por centros de interés, la importancia de la innovación y de la creatividad en el aula, el refuerzo y apoyo educativo, el aprendizaje cooperativo o el aprendizaje dialógico a través de los grupos interactivos con participación familiar (Aguilar, Alonso, Padrós y Pulido, 2010; Cardona, 2006; FernándezBatanero, 2013; Gil-del Pino, García-Fernández y Manrique-Gómez, 2017). 
Uno de los factores más influyentes para el desarrollo de prácticas inclusivas son las actitudes de los profesionales de la educación. El término "actitud" ha sido definido de diversas formas a lo largo de la literatura científica, dada la complejidad y la amplitud de este. En dicho sentido y atendiendo al ámbito educativo, la actitud se puede definir como la disposición (cognitiva, afectiva y conductual) positiva o negativa que muestran los docentes hacia los alumnos, aspecto que influye en la relación con los mismos (Dias y Cadime, 2015; Greguol, Malagodi y Carraro, 2018). Existe cierta tendencia a concebir la diversidad como un estigma o una característica negativa asociada a alumnos que presentan necesidades específicas. El mantenimiento de este tipo de actitudes por parte del profesorado puede ocultar a un conjunto de la población educativa que requiere de una atención más individualizada para reconocer y cubrir sus necesidades (Barrero-Pascuas, 2016). En este sentido, Ben-Yehuda, Leyserb y Last (2010) llevaron a cabo un trabajo en el que analizaron variables relacionadas con el éxito en los procesos formativos desarrollados por docentes con estudiantes con necesidades específicas de apoyo educativo. Los resultados señalaron que la orientación pedagógica, las actitudes hacia la inclusión, las prácticas diarias y algunas características personales como la autoeficacia percibida, influían en el éxito y la puesta en marcha de procesos educativos inclusivos.

No obstante, aunque la mayoría del profesorado admite que trabajar con este alumnado les ayuda a confiar más en sus propias habilidades como educadores, también manifiestan miedos y dudas sobre la acogida de estos alumnos en sus aulas debido, principalmente, a la falta de formación teórica y práctica con estos niños (Greguol et al., 2018). Por esto, una de las necesidades más importantes que debería ser abordada para llevar a cabo una atención temprana de calidad en las escuelas es la formación del profesorado, del equipo directivo y del resto de profesionales de la educación (Gil-del Pino et al., 2017). Es imprescindible que estos educadores reciban una formación adecuada y que su desarrollo profesional sea constante, aspecto que no solo mejoraría las actuaciones con los niños y su entorno, sino que contribuiría a la interiorización de actitudes más positivas hacia la inclusión ( $\mathrm{Hu}, \mathrm{Wu}, \mathrm{Su}$ y Roberts, 2017; Pegalajar y Colmenero, 2017; Preichardt Duek, Colombo Scarlati Domingues, Nicoletti Mizukami y Ramos Martins, 2020). Este desarrollo profesional continuo favorecería la adopción de creencias positivas acerca de la propia preparación personal, aspecto que se vincularía con la autoeficacia percibida y con la puesta en práctica de una educación inclusiva eficaz (Apablaza, 2014; Ben-Yehuda et al., 2010; Dunst y Bruder, 2014).

Otro de los elementos básicos para el desarrollo de procesos educativos inclusivos de calidad es el análisis, la investigación, la reflexión y la autoevaluación que los docentes deben hacer sobre su propia práctica (San Fabián y Granda, 2013). Siguiendo el estudio de Arnáiz y Azorín-Abellán (2014), se pudo comprobar que los docentes de las etapas de Educación Infantil y Educación Primaria, incluidos en la muestra de estudio, llevaban a cabo una adecuada programación y procesos de evaluación continua coherentes. Sin embargo, se detectaron algunas necesidades como las intervenciones de refuerzo educativo y ampliación, la coordinación entre el profesorado y la atención individualizada al alumnado. Estos resultados van en la línea de lo obtenido por Navarro-Montaño (2017) tras las entrevistas realizadas a maestros de Educación Infantil y Primaria, quienes informaban de carencias relativas a la falta de personal cualificado, de recursos materiales y humanos y a la necesidad de modificar las prácticas de aula.

Del mismo modo, atender a la diversidad implica no solo las acciones que realice el profesorado de los centros, sino también la comunicación y la colaboración que se establezca 
con otros de los principales agentes educativos en el desarrollo infantil: la familia. En este sentido, es necesario señalar que el entorno familiar contribuye a la formación en valores y a la socialización de los discentes, influyendo y participando activamente en los procesos de enseñanza-aprendizaje llevados a cabo con niños con y sin necesidades específicas de apoyo educativo (Cano-Muñoz y Martínez-González, 2019; García-Fernández, Inglés, Gonzálvez, Vicent y Gómez-Núñez, 2013).

Es necesario que los profesionales que imparten clase en la etapa de Educación Infantil asuman la responsabilidad de continuar con su desarrollo profesional y de adaptar las prácticas educativas a la amplia gama de necesidades que el alumno pueda presentar, así como de favorecer vías para una participación colaborativa entre el centro, las familias y otros servicios, aspecto fundamental para construir procesos educativos más coherentes y útiles. Según García Sánchez (2003), los objetivos o necesidades que se deberían cubrir en un futuro en la educación de los niños más pequeños comenzarían por (1) realizar intervenciones desde la prevención primaria, antes de que aparezcan los trastornos; (2) detectar tempranamente los posibles riesgos, tanto biológicos como ambientales; (3) intervenir en entornos normalizados; (4) y favorecer la coordinación entre el centro, las familias y todos los servicios que atiendan al niño desde un modelo integral de intervención.

El propósito principal de este estudio es conocer, de la mano de los educadores de la etapa de Educación Infantil, cómo se desarrolla la atención educativa a niños con necesidades específicas de apoyo educativo, examinando las estrategias, actitudes y necesidades del centro vinculadas con la inclusión. El conocimiento de estos elementos permitiría orientar los procesos de formación docente inicial y continua, así como planificar e implantar medidas que optimicen la inclusión en los centros educativos.

Por esto, el propósito general se concreta en las siguientes cuestiones de investigación:

a) ¿Qué actitudes presentan este grupo de profesionales ante la inclusión de alumnos con necesidades específicas de apoyo educativo en las aulas ordinarias?

b) ¿Qué factores influyen en el desarrollo académico y en el rendimiento del alumnado?

c) ¿Qué apoyos o recursos necesitan los centros para optimizar los procesos educativos inclusivos?

d) ¿Qué actuaciones se llevan a cabo para atender de manera individualizada al alumnado con necesidades específicas de apoyo educativo?

\section{MÉTODOLOGIA}

\subsection{PARTICIPANTES}

La muestra estuvo formada por un total de 142 maestros con edades comprendidas entre los 27 y 33 años $(M=28,93, D T=1.82)$ que imparten clase en el segundo ciclo de la etapa de Educación Infantil (3-6 años) de veinte centros públicos y concertados de Educación Infantil y Primaria españoles. La muestra se constituyó por un 52,3\% de mujeres y un 47,7\% de hombres, con una experiencia que oscilaba entre los 3 y 9 años $(M=5.14, D T=1.75)$.

Para la selección de los centros, se realizó un muestreo no probabilístico de carácter intencional, siguiendo como criterio estratégico la accesibilidad a los centros y la 
disposición a participar en el estudio. Así mismo, para seleccionar a los participantes, se llevó a cabo un muestreo no probabilístico de carácter intencional, siguiendo como criterio estratégico la selección de aquellos maestros que fuesen tutores de alguno de los distintos grupos del segundo ciclo de Educación Infantil (nivel de tres años, nivel de cuatro años o nivel de cinco años). Los participantes fueron tratados en todo momento de acuerdo con los estándares éticos de la investigación por lo que, una vez realizada la selección, se procedió a la obtención del consentimiento informado de todos y cada uno de ellos, garantizando en todo momento el anonimato y la confidencialidad de los datos.

\subsection{DISEÑO}

Siguiendo con el enfoque cuantitativo, se optó por un diseño descriptivo simple, ya que lo que se pretendía era conocer cómo se desarrollaba la atención educativa a niños con necesidades específicas de apoyo educativo en los centros, con el fin de favorecer una educación inclusiva y de calidad para estos alumnos. Además, en el instrumento de estudio, se incluyó una pregunta abierta para profundizar en el propósito de la investigación. Se considera que el hecho de mantener este tipo de preguntas en el cuestionario podría aumentar la calidad de los datos que se pudieran obtener ya que permitía, por un lado, centrar el fenómeno de estudio y, por otro, conocer las opiniones de los participantes en la investigación, más allá de las opciones incluidas en la prueba.

\subsection{INSTRUMENTOS Y VARIABLES}

Las variables estudiadas en el presente estudio fueron las actitudes y las creencias de los maestros de Educación Infantil hacia la inclusión del alumnado con necesidades específicas de apoyo educativo (rechazo, evitación, indiferencia, tolerancia y aceptación), los factores de diversidad influyentes en el rendimiento según los docentes (cultura u origen étnico, estatus socioeconómico, el talento, la capacidad, el interés de la familia, el esfuerzo de los maestros o de los niños, el clima del centro o la capacidad del colegio para adaptarse a las necesidades de su alumnado), los apoyos y/o recursos que estos profesionales consideraban necesarios para fomentar una educación inclusiva y de calidad (implicación de las familias, aumentar las vías de comunicación centro-familias, cooperación con otras instituciones, mayor formación del profesorado, recursos personales y/o materiales, coordinación entre profesionales, implicación del equipo directivo o el cambio de metodologías y prácticas) y las actuaciones que llevaban a cabo estos educadores con los alumnos.

Por esto, tras revisar diversos cuestionarios sobre actitudes, necesidades y prácticas educativas dirigidos al alumnado con necesidades específicas de apoyo educativo, se observó que los distintos instrumentos se centraban en alguno de los aspectos mencionados, pero no los incluían de manera conjunta. Debido a esto se realizó una selección de cuatro ítems del Cuestionario de Creencias y Actitudes hacia la Diferencia (Cardona, 2005). Esta adaptación estaba compuesta por dos partes diferenciadas. En la primera parte, se incluyeron cuatro preguntas acerca de los datos sociodemográficos de los participantes (edad, sexo, años de experiencia y sensibilidad hacia la diversidad), mientras que la segunda parte estuvo formada por cuatro preguntas cerradas que se contestaban a través de una escala tipo Likert con cinco opciones de respuesta. Estas preguntas cerradas hacían referencia a las actitudes hacia la diferencia, así como a los posibles recursos necesarios para ofrecer al alumnado 
con necesidades específicas de apoyo educativo una atención formativa de mayor calidad. Además, se incluyó una pregunta abierta que permitía a los participantes enumerar las distintas actuaciones que desarrollaban con este alumnado.

\subsection{PROCEDIMIENTO}

Una vez realizado el proceso de selección de la muestra de participantes y tras la obtención del consentimiento informado de los mismos, se procedió a la administración de los cuestionarios. Estos fueron distribuidos por las investigadoras en dos fases diferenciadas. En primer lugar, se repartieron de manera individual a cada uno de los participantes, junto con una carta en la que se indicaban las instrucciones para completar la prueba y se les agradecía su participación en el proyecto, con el fin de facilitar la tarea a los sujetos y motivarlos a la hora de contestar. En segundo lugar, tanto los cuestionarios como las instrucciones fueron enviados por correo electrónico, con el fin de proporcionar una vía alternativa y facilitar la entrega de estos.

Una vez completadas las pruebas, se agradeció la implicación de todos y cada uno de los participantes a través de una carta por correo electrónico indicando, además, que se les ofrecerían los resultados una vez que hubiese concluido el estudio. De este modo, se pretendía que los docentes obtuvieran un conocimiento más completo de la realidad educativa, brindándoles así la oportunidad de conocer otras prácticas y herramientas que pudieran ayudarles a solventar las distintas necesidades de sus centros educativos. De esta manera, una vez que se analizaron los datos, las investigadoras enviaron los resultados a través del correo electrónico a cada uno de los participantes, ofreciendo su ayuda para la comprensión de estos.

\subsection{ANÁLISIS DE LOS DATOS}

El análisis de datos cuantitativo se llevó a cabo a través del paquete estadístico SPSS 23. Para las preguntas número 1, 2, 3 y 4 se calcularon los promedios (medias) y desviaciones típicas. Además, también se realizó un análisis de frecuencias y de porcentajes. El análisis de datos cualitativo de la pregunta número 5 se llevó a cabo a través de un análisis del contenido de las respuestas, de manera que, posteriormente, se reagruparon los contenidos en categorías, empleando para esto el paquete estadístico AQUAD 7.

\section{RESULTADOS}

\subsection{ACTITUDES HACIA LA DIVERSIDAD}

En la Tabla 1 pueden observarse los resultados correspondientes a la primera pregunta del cuestionario, que hacía referencia a la percepción general que el profesorado tenía sobre las actitudes de sus compañeros de trabajo, hacia la inclusión de alumnos con necesidades específicas de apoyo educativo. 
Tabla 1. Percepción general del profesorado sobre las actitudes de sus compañeros, hacia la inclusión de alumnos con necesidades específicas de apoyo educativo

\begin{tabular}{|c|c|c|c|c|c|c|c|}
\hline & & & Rech. & Evit. & Indi. & Tol. & Acep. \\
\hline & $M$ & $D T$ & $\%$ & $\%$ & $\%$ & $\%$ & $\%$ \\
\hline 1. Necesidades educativas especiales & 4.43 & .94 & 0 & 7 & 7 & 22 & 64 \\
\hline 2. Altas capacidades & 4.36 & .84 & 0 & 0 & 21 & 22 & 57 \\
\hline 3. Dificultades específicas de aprendizaje & 4.70 & .43 & 0 & 0 & 0 & 21 & 79 \\
\hline 4. Condiciones personales o de historia escolar & 4.64 & .63 & 0 & 0 & 7 & 22 & 71 \\
\hline 5. Conducta disruptiva ${ }^{1}$ & 4.64 & .50 & 0 & 0 & 0 & 36 & 64 \\
\hline 6. Integración tardía & 4.64 & .50 & 0 & 0 & 0 & 36 & 64 \\
\hline
\end{tabular}

${ }^{1}$ Los trastornos graves de la conducta se incluyen, según la legislación española actual, dentro del grupo con necesidades educativas especiales. Sin embargo, debido a los propósitos de esta investigación, los alumnos con conductas disruptivas fueron considerados como una categoría distinta (aunque no estuviesen diagnosticados como trastornos graves de la conducta).

Nota : Rech. = Rechazo, Evit. = Evitación, Indi. = Indiferencia, Tol. = Tolerancia, Acep. = Aceptación.

Como se puede observar, los encuestados percibían actitudes muy favorables hacia la diversidad en sus compañeros $(M=4.56)$. Destacaron las actitudes favorables hacia el alumnado con dificultades específicas de aprendizaje $(M=4.70, D T=.43)$, mientras que las actitudes hacia el alumnado con altas capacidades también fueron positivas, pero en menor medida que las anteriores $(M=4.36, D T=.84)$. De hecho, la mayoría de los encuestados (79\%) escogieron la "aceptación" como la actitud que mejor definía el modo en el que su centro respondía al alumnado con dificultades específicas de aprendizaje, frente al 57\% que también escogió la "aceptación" como la actitud que mejor definía la atención al alumnado con altas capacidades. Únicamente el 7\% de los participantes eligieron la "evitación" como la forma en la que sus centros respondían a las necesidades educativas especiales.

En la Tabla 2 se recogen los datos de la segunda pregunta del cuestionario, referente a la actitud que los propios participantes, como maestros de Educación Infantil, asumían a la hora de atender al alumnado con necesidades específicas de apoyo educativo.

Tabla 2. Actitudes del profesorado hacia la inclusión del alumnado con necesidades específicas de apoyo educativo

\begin{tabular}{|l|c|c|c|c|c|c|c|}
\hline & & & Rech. & Evit. & Indi. & Tol. & Acep. \\
\hline & $M$ & $D T$ & $\%$ & $\%$ & $\%$ & $\%$ & $\%$ \\
\hline 1. Necesidades educativas especiales & 4.93 & .27 & 0 & 0 & 0 & 7 & 93 \\
\hline 2. Altas capacidades & 4.86 & .53 & 0 & 0 & 0 & 7 & 93 \\
\hline 3. Dificultades académicas & 4.93 & .27 & 0 & 0 & 0 & 7 & 93 \\
\hline 4. Condiciones personales o de historia escolar & 4.71 & .61 & 0 & 0 & 7 & 15 & 78 \\
\hline 5. Conducta disruptiva & 4.93 & .27 & 0 & 0 & 0 & 7 & 93 \\
\hline 6. Integración tardía & 4.93 & .27 & 0 & 0 & 0 & 7 & 93 \\
\hline
\end{tabular}

Nota : Rech. $=$ Rechazo, Evit. = Evitación, Indi. = Indiferencia, Tol. = Tolerancia, Acep.= Aceptación. 
Los maestros entrevistados se describieron a sí mismos con actitudes muy favorables hacia la atención del alumnado con necesidades específicas de apoyo educativo $(M=$ 4.88). No obstante, destacaron las actitudes positivas hacia el alumnado con necesidades educativas especiales $(M=4.93, D T=.27)$, alumnado con dificultades específicas de aprendizaje $(M=4.93, D T=.27)$, alumnado con conductas disruptivas (véase nota 1 al final del documento) $(M=4,93, D T=.27)$ y hacia el alumnado con necesidades por integración tardía $(M=4.93, D T=.27)$. La media más baja fue la obtenida para el alumnado con necesidades por condiciones personales o de historia escolar $(M=4.71, D T=.61)$, aunque las actitudes también resultaron favorables. Según la opinión del $93 \%$ del profesorado, la "aceptación" era la actitud que predominaba a la hora de atender al alumnado con necesidades educativas especiales, con altas capacidades, con dificultades específicas de aprendizaje y con necesidades derivadas de su conducta disruptiva e integración tardía. El $78 \%$ de estos docentes también escogió la "aceptación" como la actitud que mejor definía su actuación con el alumnado con necesidades por condiciones personales o de historia escolar. Además, solo el 7\% eligieron la "indiferencia" como la actitud que mantenían hacia el alumnado con dificultades por condiciones personales o de historia escolar.

\subsection{FACTORES INFLUYENTES EN EL RENDIMIENTO DEL ALUMNADO}

En la Tabla 3 se muestran los resultados derivados de la tercera cuestión, que aludía a diversos factores que podrían influir en el rendimiento del alumnado.

Tabla 3. Factores influyentes en el rendimiento del alumno

\begin{tabular}{|l|c|c|c|c|c|c|c|}
\hline & & $\begin{array}{c}\text { Muy en } \\
\text { desacuerdo }\end{array}$ & Desacuerdo & Neutral & $\begin{array}{c}\text { De } \\
\text { acuerdo }\end{array}$ & $\begin{array}{c}\text { Muy de } \\
\text { acuerdo }\end{array}$ \\
\hline $\begin{array}{l}\text { Su cultura u origen } \\
\text { étnico }\end{array}$ & 3.36 & .63 & 0 & 7 & 50 & 43 & 0 \\
\hline $\begin{array}{l}\text { El estatus } \\
\text { socioeconómico }\end{array}$ & 3.36 & .63 & 0 & 7 & 50 & 43 & 0 \\
\hline El talento & 3.50 & .85 & 0 & 7 & 50 & 29 & 14 \\
\hline La capacidad & 3.79 & 1.25 & 0 & 29 & 0 & 35 & 36 \\
\hline El interés de la familia & 4.93 & .27 & 0 & 0 & 0 & 7 & 93 \\
\hline $\begin{array}{l}\text { El esfuerzo de los } \\
\text { maestros }\end{array}$ & 4.93 & .27 & 0 & 0 & 0 & 7 & 93 \\
\hline $\begin{array}{l}\text { El esfuerzo de los } \\
\text { niños }\end{array}$ & 4.64 & .84 & 0 & 7 & 0 & 14 & 79 \\
\hline El clima del centro & 4.21 & .80 & 0 & 0 & 21 & 35 & 43 \\
\hline $\begin{array}{l}\text { Adaptación del cen- } \\
\text { tro a las necesidades }\end{array}$ & 4.29 & 1.07 & 7 & 0 & 0 & 43 & 50 \\
\hline
\end{tabular}


Tal y como muestran los resultados, los participantes otorgaron una importancia elevada a la mayoría de los posibles factores influyentes en el rendimiento, sobre todo a aquellos que tenían que ver con el contexto en el que se desarrolla el proceso educativo de los discentes $(M=4.11)$. De este modo, según este grupo de docentes, los factores más influyentes en el rendimiento serían el interés de la familia $(M=4.93, D T=.27)$ y el esfuerzo realizado por los maestros $(M=4.93, D T=.27)$. Tras estos factores se situaron otros elementos con un nivel de influencia menor como el talento $(M=3.50, D T=.85)$, el estatus socioeconómico $(M=3.36, D T=.63)$ y el origen cultural $(M=3.36, D T=.63)$. Estos datos reflejaron que, la mayoría de los encuestados (93\%), opinaban que el esfuerzo de los docentes y el interés de la familia eran los factores más influyentes en el rendimiento, mientras que únicamente el $14.3 \%$ opinaban que era el talento. Además, el 29\% estaban en desacuerdo a la hora de considerar la capacidad de los alumnos como un factor influyente en su rendimiento.

\subsection{APOYOS Y RECURSOS NECESARIOS PARA ATENDER A LA DIVERSIDAD}

En la Tabla 4 se muestran los resultados concernientes a la cuarta pregunta, que hacía referencia al tipo de apoyos y recursos necesarios en los centros para atender de manera adecuada a la diversidad del alumnado.

Tabla 4. Apoyos y recursos necesarios para la mejora de la atención a la diversidad del alumnado

\begin{tabular}{|l|c|c|c|c|c|c|c|}
\hline & & & Nada & Algo/ poco & Indeciso & Bastante & Mucho \\
\hline & $M$ & $D T$ & $\%$ & $\%$ & $\%$ & $\%$ & $\%$ \\
\hline Implicación de las familias & 3.43 & 1.40 & 7 & 21 & 29 & 7 & 36 \\
\hline $\begin{array}{l}\text { Aumentar las vías de } \\
\text { comunicación entre el centro y } \\
\text { las familias }\end{array}$ & 3.36 & 1.34 & 7 & 21 & 29 & 14 & 29 \\
\hline $\begin{array}{l}\text { Cooperación con otras } \\
\text { instituciones }\end{array}$ & 3.21 & .89 & 0 & 21 & 43 & 29 & 7 \\
\hline $\begin{array}{l}\text { Mayor formación del } \\
\text { profesorado }\end{array}$ & 3.00 & .88 & 0 & 29 & 50 & 14 & 7 \\
\hline $\begin{array}{l}\text { Disponer de más recursos } \\
\text { materiales }\end{array}$ & 4.43 & .65 & 0 & 0 & 7 & 43 & 50 \\
\hline $\begin{array}{l}\text { Disponer de más recursos } \\
\text { humanos }\end{array}$ & 4,71 & .47 & 0 & 0 & 0 & 29 & 71 \\
\hline Coordinación entre profesionales & 3.07 & 1.49 & 29 & 0 & 21 & 36 & 14 \\
\hline Implicación del equipo directivo & 2.79 & 1.19 & 7 & 43 & 29 & 7 & 14 \\
\hline $\begin{array}{l}\text { Cambio de metodologías y } \\
\text { prácticas }\end{array}$ & 3.00 & 1.04 & 0 & 36 & 43 & 7 & 14 \\
\hline
\end{tabular}

Tras el análisis de los resultados, las necesidades más importantes, según los docentes encuestados, eran el aumento de recursos humanos $(M=4.71, D T=.47)$ y el aumento de 
recursos materiales $(M=4.43, D T=.65)$. Así mismo, también se consideraron importantes el aumento de la implicación familiar $(M=3.43, D T=1.40)$, el incremento de las vías de comunicación entre el centro y las familias $(M=3.36, D T=1.34)$ y la cooperación con otras instituciones $(M=3.21, D T=.89)$. Las necesidades con puntuaciones menores, pero que también deben ser tenidas en cuenta, fueron la coordinación entre los profesionales del centro $(M=3.07, D T=1.49)$, el aumento de la formación del profesorado $(M=3.00, D T$ $=.88)$, el cambio de metodologías y prácticas $(M=3.00, D T=1.04)$ y la implicación del equipo directivo en último lugar $(M=2.79, D T=1.19)$. La mayoría de los participantes (71\%) afirmaron que el incremento de recursos materiales era la principal necesidad a abordar para la mejora de la calidad en la atención a la diversidad, seguida del 50\% que escogieron el aumento de recursos materiales. Según los encuestados, las necesidades que presentaban una menor importancia eran la cooperación con otras instituciones y la mejora de la formación del profesorado (7\%). Además, el $43 \%$ opinaba que la implicación del equipo directivo era poco importante, mientras que el $29 \%$ no otorgó ningún tipo de importancia a la coordinación entre los profesionales que componen el centro a la hora de atender a la diversidad.

\subsection{ACTUACIONES PARA LA ATENCIÓN INDIVIDUALIZADA AL ALUMNADO CON NECESIDADES ESPECÍFICAS DE APOYO EDUCATIVO}

En la Tabla 5 se muestran los resultados extraídos de la quinta pregunta, de carácter abierto, que permitía a los docentes enumerar las actuaciones que llevaban a cabo con el alumnado con necesidades específicas de apoyo educativo.

Tabla 5. Medidas de atención a la diversidad

\begin{tabular}{|l|l|}
\hline Necesidad específica de apoyo educativo & \multicolumn{1}{|c|}{ Estrategias } \\
\hline Integración tardía & $\begin{array}{l}\text { 1. Organización de talleres en el aula. } \\
\text { 2. Actividades de refuerzo (con ilustraciones). } \\
\text { 3. Reuniones periódicas con las familias. } \\
\text { 4. Coordinación con profesionales internos y externos } \\
\text { al centro. } \\
\text { 5. Trabajo en el aula a través de rincones. }\end{array}$ \\
\hline Condiciones personales o de historia & $\begin{array}{l}\text { 1. Reuniones con las familias para establecer pautas } \\
\text { educativas comunes y prestar la ayuda que sea } \\
\text { necesaria. } \\
\text { 2. Grupos interactivos con participación familiar. } \\
\text { 3. Refuerzo en el aula. }\end{array}$ \\
\hline Necesidades educativas especiales & $\begin{array}{l}\text { 1. Coordinación con otros profesionales (tanto } \\
\text { internos como externos al centro). } \\
\text { 2. Adaptaciones del currículo. } \\
\text { 3. Adaptaciones en tiempos, materiales y espacios. } \\
\text { 4. Co-enseñanza. }\end{array}$ \\
\hline
\end{tabular}




\begin{tabular}{|l|l|}
\hline Altas capacidades & $\begin{array}{l}\text { 1. Actividades de ampliación. } \\
\text { 2. Enriquecimiento en el aula. } \\
\text { 3. Tutoría entre iguales. }\end{array}$ \\
\hline Dificultades específicas de aprendizaje & $\begin{array}{l}\text { 1. Repaso continuo de contenidos y refuerzo en los } \\
\text { aprendizajes. } \\
\text { 2. Co-enseñanza. } \\
\text { 3. Trabajo en rincones. }\end{array}$ \\
\hline Conducta disruptiva & $\begin{array}{l}\text { 1. Reforzamiento positivo. } \\
\text { 2. Reuniones con las familias. } \\
\text { 3. Economía de fichas. }\end{array}$ \\
\hline
\end{tabular}

Como se puede observar, las prácticas más empleadas por este grupo de maestros de Educación Infantil eran el establecimiento de reuniones periódicas con las familias para aunar pautas educativas, el trabajo a través de rincones en el aula, la co-enseñanza, la coordinación con otros profesionales internos o externos al centro que intervengan en la educación del alumnado, las actividades de refuerzo en el aula y el refuerzo emocional para lograr motivar al alumnado. No obstante, aunque estas son las medidas más empleadas, según este grupo de docentes también se llevaban a cabo otras dependiendo de la necesidad. Así, para las necesidades derivadas de la integración tardía del alumnado, los docentes realizaban talleres en los que se implicaba a las familias; para las necesidades debidas a condiciones personales o de historia escolar resultaba beneficioso la creación de grupos interactivos con participación familiar; las adaptaciones curriculares, de espacios, tiempos y materiales se llevaron a cabo con alumnado con necesidades educativas especiales; para las necesidades derivadas de altas capacidades, los docentes emplearon las actividades de ampliación, de enriquecimiento y la tutoría entre iguales; mientras que para las necesidades surgidas por conductas disruptivas se llevaron a cabo programas de economía de fichas.

\section{DISCUSIÓN Y CONCLUSIONES}

Las intervenciones educativas de carácter inclusivo con niños con necesidades específicas de apoyo educativo en la etapa de Educación Infantil se consolidan de manera progresiva como una de las actuaciones más útiles a corto y largo plazo, por su eficacia, efectos y carácter preventivo-educativo (Amar, 2015). Por esto, el propósito principal del presente trabajo se centraba en conocer, según las opiniones de los educadores de la etapa de Educación Infantil, cómo se desarrollaba en los colegios la atención educativa a niños con necesidades específicas de apoyo educativo, examinando las prácticas, actitudes del profesorado y necesidades del centro vinculadas con la inclusión, con el fin de favorecer una educación de calidad para todos los alumnos. Este objetivo general se concretaba en un conjunto de cuestiones de investigación a las que se pretendía dar respuesta tras la recogida y análisis de los datos.

La primera cuestión de investigación hacía referencia a las actitudes que presentaban los participantes ante la inclusión en las aulas ordinarias de alumnos con necesidades específicas de apoyo educativo. Así, tras el análisis de las dos primeras preguntas del 
cuestionario se observó que, de manera general, estos maestros señalaron que tanto sus compañeros, como ellos mismos, mantienen actitudes muy favorables hacia la diversidad de su alumnado, predominando la aceptación y la tolerancia frente a la indiferencia, el rechazo o la evitación. Estos resultados son coherentes con investigaciones previas que revelaban la importancia de las actitudes del profesorado para el desarrollo de procesos de inclusión educativa de calidad (Ben-Yehuda et al., 2010; Greguol et al., 2018). No obstante, cabe indicar que el profesorado se describe a sí mismo con actitudes más favorables que las que atribuyen a sus compañeros, es decir, las personas suelen percibirse de una manera más positiva comparada con la visión que tienen de los demás. Estos resultados pueden ser explicados por la autoeficacia percibida, elemento que favorecería la adopción de prácticas inclusivas como una forma de expresión de las actitudes positivas hacia el alumnado con necesidades específicas de apoyo educativo (Ben-Yehuda et al., 2010; Dias y Cadime, 2015; Dunst y Bruder, 2014). En el caso de la percepción que tienen sobre las actitudes de los demás, establecen que estas son más favorables hacia el alumnado con necesidades educativas derivadas de sus dificultades específicas de aprendizaje mientras que, en el caso de la descripción de sus propias actitudes, estas son más positivas hacia el alumnado con necesidades educativas especiales, dificultades específicas de aprendizaje, integración tardía o conducta disruptiva. En este sentido, es importante orientar la formación básica y continua del profesorado hacia la práctica con alumnos con necesidades derivadas de otros factores distintos a los mencionados, con el fin de fomentar el desarrollo de actitudes positivas hacia todos los alumnos (Apablaza, 2014; Hu et al., 2017; Preichardt Duek et al., 2020).

En cuanto a la segunda cuestión de investigación, referida a los factores más influyentes en el desarrollo académico del alumnado, los análisis revelan que, según los encuestados, el interés de la familia y el esfuerzo de los maestros son los dos aspectos más decisivos en el progreso escolar de los niños. Estos datos concuerdan con otras investigaciones en las que se establecía que, para favorecer el desarrollo infantil, era necesario plantear actuaciones no solo con el propio alumno, sino también con las familias y con el entorno, como aspectos implicados en la conformación de la persona (Cano-Muñoz y Martínez-González, 2019; García-Fernández et al., 2013). Además, siguiendo en esta línea de investigación, puede afirmarse que el hecho de abogar por una perspectiva más preventiva, inclusiva y educativa revela que los educadores deberán adaptar sus prácticas, concediendo una gran importancia a los elementos contextuales (familia y colegio) a la hora de propiciar el desarrollo integral del niño (Giné et al., 2016; Aboes Vercelli et al., 2019).

Con la tercera pregunta de investigación se analizaba qué tipo de apoyos y recursos asociados a la atención educativa del alumnado con necesidades específicas de apoyo educativo eran necesarios para promover una educación inclusiva. Los resultados mostraron que el aumento tanto de recursos materiales, como de recursos humanos, son los principales medios para la mejora de la atención educativa a la diversidad del alumnado (NavarroMontaño, 2017). Por el contrario, la formación del profesorado que, según otros estudios, era considerado como uno de los elementos fundamentales para atender adecuadamente a la diversidad y para la construcción de actitudes positivas (Greguol et al., 2018; Hu et al., 2017; Pegalajar y Colmenero, 2017; Preichardt Duek et al., 2020), en esta investigación queda relegado a un segundo plano. Las actitudes positivas hacia la diversidad, junto con la autoeficacia percibida podría hacer que los docentes no aprecien la necesidad de una mayor preparación (Ben-Yehuda et al., 2010; Dunst y Bruder, 2014), sino que atribuyan 
las carencias detectadas a una falta de recursos materiales y personales. La autoevaluación que los docentes deben hacer sobre su propia práctica resulta fundamental para la detección de necesidades y la puesta en marcha de intervenciones ajustadas a las características del alumnado (Arnáiz y Azorín-Abellán, 2014; San Fabián y Granda, 2013).

Por último, con la cuarta cuestión de investigación, se pretendía conocer qué tipo de actuaciones se llevaban a cabo para atender de manera individualizada al alumnado con necesidades específicas de apoyo educativo. Los datos señalan que las estrategias más empleadas por este grupo de docentes son el establecimiento de reuniones periódicas con las familias para aunar pautas educativas, el trabajo a través de rincones en el aula, la co-enseñanza, la coordinación con otros profesionales internos y externos al centro que intervienen en la educación del alumnado, las actividades de refuerzo en el aula y el refuerzo emocional para lograr motivar al alumnado, tal y como se recoge en otras investigaciones previas (Aguilar et al., 2010; Arnáiz y Azorín-Abellán, 2014; Cardona, 2006; Cano-Muñoz y Martínez-González, 2019; Fernández-Batanero, 2013; GarcíaFernández et al., 2013; Gil-del Pino et al., 2017; Greguol et al., 2018). Además, junto con estas medidas, también se llevan a cabo otras dependiendo de la necesidad. Así, para las que son derivadas de la integración tardía del alumnado, los docentes incluían la puesta en marcha de talleres en los que se implicaba a las familias; para las necesidades derivadas de condiciones personales o de historia escolar, resultaba beneficioso la creación de grupos interactivos con participación familiar; para el alumnado con necesidades educativas especiales se realizaban adaptaciones curriculares, de espacios, tiempos y materiales; para las necesidades derivadas de altas capacidades, los docentes llevaban a cabo actividades de ampliación, actividades de enriquecimiento y la tutoría entre iguales; mientras que para las necesidades surgidas por las conductas disruptivas se planteaban programas de economía de fichas. La aplicación de medidas inclusivas para atender a la diversidad del alumnado, no solo a aquellos que se encuentran en riesgo por sus necesidades específicas de apoyo educativo, resulta imprescindible para aumentar la motivación de los niños, mejorar su implicación emocional, favorecer el desarrollo de una atención individualizada, así como para promover vías de comunicación entre los alumnos, los docentes y las familias (Aguilar et al., 2010; Balongo-González y Mérida-Serrano, 2016; Navarro-Montaño, 2017), lo que impulsa la creación de centros que se definan como verdaderas comunidades de aprendizaje.

Los resultados obtenidos se interpretan valorando algunas limitaciones que deberían ser tenidas en cuenta en futuras investigaciones en este campo de estudio. En primer lugar, cabe destacar la selección de la muestra a propósito, lo que podría conllevar algunos sesgos como la dificultad de generalizar los resultados a una población más amplia. En segundo lugar, en lo relativo al instrumento de investigación, se emplearon únicamente cinco cuestiones principales, a partir de la adaptación del cuestionario elaborado por Cardona (2005). En tercer lugar, no se tuvieron en cuenta las posibles diferencias entre el profesorado de centros públicos y concertados-privados. Por último, no se valoró la opinión de las familias en cuanto a la atención que sus hijos reciben. Para futuras investigaciones se podrían plantear aspectos como (1) aumentar el tamaño de la muestra y realizar una selección al azar, (2) incluir nuevas cuestiones o ítems en el cuestionario aplicado, analizando las propiedades psicométricas del mismo, (3) realizar un estudio comparativo entre colegios públicos y concertados-privados e (4) incluir la visión de las familias.

A pesar de las limitaciones y consideraciones posteriores, este estudio ha permitido obtener un conocimiento más exhaustivo sobre los procesos educativos que se llevan a 
cabo en la Educación Infantil, así como de las actitudes y necesidades detectadas por el profesorado de esta etapa educativa. Por esto, tras los resultados obtenidos se puede afirmar que las actitudes generales del profesorado hacia la inclusión de niños con necesidades específicas de apoyo educativo en Educación Infantil son positivas, lo que puede considerarse como un factor fundamental a la hora de desarrollar prácticas inclusivas. Además, este grupo de profesionales considera las variables contextuales (familia y profesorado) como elementos esenciales a tener en cuenta en el óptimo desarrollo de los niños, demandando, también, un mayor número tanto de profesionales, como de materiales para poder atender de manera adecuada al conjunto del alumnado.

La realización de intervenciones preventivas, la detección temprana de las necesidades, la intervención en entornos normalizados, la coordinación entre los distintos agentes educativos y la autoevaluación docente son factores básicos para el desarrollo de una adecuada atención a la diversidad (García-Sánchez, 2003; San Fabián y Granda, 2013). Del mismo modo, la adopción de un modelo educativo inclusivo implica la creación de espacios de diálogo en los que se fomenten actitudes positivas hacia todo el alumnado (Ainscow, 2012), de forma que se evite la falta de reconocimiento de un conjunto de la población educativa que precisa de una atención más individualizada por sus necesidades específicas (Barrero-Pascuas, 2016). La implementación de prácticas educativas en las que se tiene en cuenta a la totalidad del alumnado no solo favorece el desarrollo de todos, sino que también promueve la mejora de las relaciones interpersonales del alumnado y de su competencia escolar, lo que transciende en la construcción de una sociedad más justa.

\section{REFERENCIAS BIBLIOGRÁFICAS}

Aboes Vercelli, L. d. C., Alcãntara, C. R. y Silveira Barbosa, M. C. (2019). Learning and development in children education: Contributions of the national curricular guidelines and the common national curricular base for this discussion. Dialogia, (31), 33-43. doi:10.5585/dialogia.N31.11455

Aguilar, C., Alonso, M. J., Padrós, M. y Pulido, M. A. (2010). Lectura dialógica y transformación en las Comunidades de Aprendizaje. Revista Interuniversitaria de Formación del Profesorado, 24(1), 31-44.

Ainscow, M. (2012). Haciendo que las escuelas sean más inclusivas: lecciones a partir del análisis de la investigación internacional. Revista de Inclusión Educativa, 5(1), 39-49.

Amar, J. (2015). Desarrollo infantil y prácticas de cuidado. Barranquilla: Universidad del Norte.

Apablaza, M. (2014). Representaciones sociales de profesores respecto de la diversidad escolar en relación a los contextos de desempeño profesional, prácticas y formación inicial. Estudios Pedagógicos, 40(1), 7-24. doi: http://dx.doi.org/10.4067/S0718-07052014000100001.

Arnáiz, P. y Azorín-Abellán, C. M. (2014). Autoevaluación docente para la mejora de los procesos educativos en escuelas que caminan hacia la inclusión. Revista Colombiana de Educación, (67), 227-245.

Balongo-González, E. y Mérida-Serrano, R. (2016). El clima de aula en los proyectos de trabajo. Crear ambientes de aprendizaje para incluir la diversidad infantil. Perfiles educativos, 38(152), 146-162.

Barrero-Pascuas, A. M. (2016). Lo visible e invisible de la diversidad en la educación infantil. Infancias Imágenes, 15(2), 262-270. doi: 10.14483/udistrital.jour.infimg.2016.2.a07

Barton, E. E. y Smith, B. J. (2015). Advancing high-quality preschool inclusion: a discussion and recommendations for the field. Topis in Early childhood Special Education, 35(2), 69-78. doi: $10.1177 / 0271121415583048$

Ben-Yehuda, S., Leyserb, Y. y Last, U. (2010). Teacher educational beliefs and sociometric status 
of special educational needs (SEN) students in inclusive classrooms. International Journal of Inclusive Education, 14(1), 17-34. doi: http://dx.doi.org/10.1080/13603110802327339

Cano-Muñoz, M. A. y Martínez-González, R. A. (2019). Relación entre centros escolares y familias: responsabilidades compartidas para un proceso de enseñanza-aprendizaje eficaz. En C. J. Inglés, C. Ruiz-Esteban y M. S. Torregrosa (Coords.), Manual para Psicólogos Educativos: Teoría y prácticas (pp. 227-259). Madrid: Pirámide.

Cardona, M. C. (2005). Creencias, percepciones y actitudes hacia la inclusión: una síntesis de la literatura de investigación. En M. C. Jiménez (coord.), Pedagogía Diferencial. Diversidad y Equidad (pp. 239-266). Madrid: Pearson Educación.

. (2006). Diversidad y Educación Inclusiva: enfoques metodológicos y estrategias para una enseñanza colaborativa. Madrid: Pearson-Prentice Hall.

Dias, P. C. y Cadime, I. (2015). Effects of personal and professional factors on teachers' attitudes towards inclusion in preschool. European Journal of Special Needs Education, 31(1), 111-123. doi: 10.1080/08856257.2015.1108040

Dunst, C. J. y Bruder, M. B. (2014). Preservice professional preparation and teachers' self-efficacy appraisals of natural environment and inclusion practices. Teacher Education and Special Education, 37(2), 121-132. doi: 10.1177/0888406413505873

Fernández-Batanero, J. M. (2013). Competencias docentes y educación inclusiva. Revista Electrónica de Investigación Educativa, 15(2), 82-99. Recuperado de http://redie.uabc.mx

García-Fernández, J. M., Inglés, C. J., Gonzálvez, C., Vicent, M. y Gómez-Núñez, M. I. (2013). Familia y discapacidad intelectual: un estudio bibliométrico de 2000-2011 a través de la Social Science Citation Index. Siglo Cero, 44(2), 63-78.

García-Sánchez, F. A. (2003). Objetivos de futuro de la Atención Temprana. Revista de Atención Temprana, 6(1), 32-37.

Gil-del Pino, M. C., García-Fernández, C. M. y Manrique-Gómez, M. A. (2017). El poder de las expectativas del docente en el logro académico de los escolares inmigrantes. Revista de Pedagogía, 38(102), 97-121. Recuperado de http://www.redalyc.org/articulo.oa?id=65952814006

Giné, C., Balcells-Balcells, A., Cañadas, M. y Paniagua, G. (2016). Early childhood inclusion in Spain. Infants \& Young Children, 29(3), 223-230. doi: 10.1097/IYC0000000000000068

Greguol, M., Malagodi, B. M. y Carraro, A. (2018). Inclusion of students with disabilities in physical education classes: teachers' attitudes in regular schools. Revista Brasileira de Educação Especial, 24(1), 33-44. doi: http://dx.doi.org/10.1590/S1413-65382418000100004

Hu, B. Y., Wu, H. P., Su, X. Y. y Roberts, S. K. (2017). An examination of chinese preservice and inservice early childhood teachers' perspectives on the importance and feasibility of the implementation of key characteristics of quality inclusion. International Journal of Inclusive Education, 21(2), 187-204. doi:10.1080/13603116.2016.1193563

Navarro-Montaño, M. J. (2017). Mejorar la calidad de la educación inclusiva: propuesta de indicadores. Revista de Pedagogía, 38(102), 122-143. Recuperado de http://www.redalyc.org/ articulo.oa?id=65952814007

Odom, S. L., Buysse, V. y Soukakou, E. (2011). Inclusion for young children with disabilities: A quarter century of research perspectives. Journal of Early Intervention, 33(4), 344-356. doi: https://doi.org/10.1177/1053815111430094

Pegalajar, M. C. y Colmenero, M. J. (2017). Actitudes y formación docente hacia la inclusión en Educación Secundaria Obligatoria. Revista Electrónica de Investigación Educativa, 19(1), 8497. doi: https://doi.org/10.24320/redie.2017.19.1.765

Preichardt Duek, V., Colombo Scarlati Domingues, I. M., Nicoletti Mizukami, M. d. G. y Ramos Martins, L. d. A. (2020). Continuing teacher training for inclusive education: An experience with teaching cases. Revista Ibero-Americana De Estudos Em Educacao, 15, 916-+. doi:10.21723/ riaee.v15i5.13508

San Fabián, J. L. y Granda, A. (2013). Autoevaluación de centros educativos. Madrid: Síntesis. 
